\title{
Lamina propria macrophage phenotypes in relation to Escherichia coli in Crohn's disease
}

\author{
Timothy R. Elliott ${ }^{1,2,5^{*}+}$, Neil B. Rayment ${ }^{1 \dagger}$, Barry N. Hudspith ${ }^{1}$, Rebecca E. Hands ${ }^{4}$, Kirstin Taylor ${ }^{2,3}$, \\ Gareth C. Parkes ${ }^{1,2}$, Natalie J. Prescott ${ }^{3}$, Liljana Petrovska', John Hermon-Taylor ${ }^{1}$, Jonathan Brostoff', \\ Alex Boussioutas ${ }^{5}$, Christopher G. Mathew ${ }^{3}$, Stephen A. Bustin ${ }^{6}$ and Jeremy D. Sanderson ${ }^{1,2}$
}

\begin{abstract}
Background: Abnormal handling of E. coli by lamina propria (LP) macrophages may contribute to Crohn's disease (CD) pathogenesis. We aimed to determine LP macrophage phenotypes in CD, ulcerative colitis (UC) and healthy controls $(\mathrm{HC})$, and in $C D$, to compare macrophage phenotypes according to E. coli carriage.

Methods: Mucosal biopsies were taken from 35 patients with CD, 9 with UC and 18 HCs. Laser capture microdissection was used to isolate $E$. coli-laden and unladen LP macrophages from ileal or colonic biopsies. From these macrophages, mRNA was extracted and cytokine and activation marker expression measured using RT-qPCR.

Results: E. coli-laden LP macrophages were identified commonly in mucosal biopsies from CD patients $(25 / 35,71 \%)$, rarely in UC $(1 / 9,11 \%)$ and not at all in healthy controls (0/18). LP macrophage cytokine mRNA expression was greater in CD and UC than healthy controls. In CD, E. coli-laden macrophages expressed high IL-10 \& CD163 and lower TNFa, IL-23 \& iNOS irrespective of macroscopic inflammation. In inflamed tissue, E. coli-unladen macrophages expressed high TNFa, IL-23 \& iNOS and lower IL-10 \& CD163. In uninflamed tissue, unladen macrophages had low cytokine mRNA expression, closer to that of healthy controls.
\end{abstract}

Conclusion: In CD, intra-macrophage E. coli are commonly found and LP macrophages express characteristic cytokine mRNA profiles according to E. coli carriage. Persistence of E. coli within LP macrophages may provide a stimulus for chronic inflammation.

Keywords: Crohn's disease, Escherichia coli, Macrophages

\section{Background}

The exact pathogenesis of Crohn's disease (CD) remains unclear, but it is likely to result from a dysfunctional interaction between components of the intestinal microbiota and an abnormal innate immune system and mucosal barrier [1,2].

Potential roles for numerous bacteria in $\mathrm{CD}$ pathogenesis have been investigated [3-5], but none have been shown to be clearly causative. A possible role for $E$. coli has been highlighted by the isolation of a pathogenic subset labelled adherent-invasive E. coli (AIEC) from the mucosa in some

\footnotetext{
* Correspondence: elliott_timothy@hotmail.com

${ }^{\dagger}$ Equal contributors

'Diabetes and Nutritional Sciences Division, King's College London, Franklin Wilkins Building, 150 Stamford Street, London SE1 9NH, UK

2Department of Gastroenterology, Guy's and St Thomas' NHS Foundation

Trust, St Thomas' Hospital, London SE1 7EH, UK

Full list of author information is available at the end of the article
}

patients with $C D[6,7]$. Studies show that AIEC survive and replicate within macrophages in vitro $[8,9]$ and that $E$. coli can be isolated from lamina propria (LP) macrophages in $C D[5,10,11]$. This has led to the hypothesis that $E$. coli may cross the mucosal barrier and persist within LP macrophages as a stimulus for chronic inflammation in $\mathrm{CD}$. Of note, most studies report that AIEC are isolated in less than half of $C D$ cases $[6,7]$ and other less pathogenic E. coli can also be recovered by intracellular culture in CD $[6,12]$. In addition, recent data demonstrates that macrophages may be dysfunctional in CD [1], and so it is likely that both bacterial and host factors contribute to intra-macrophage bacterial persistence in CD.

Human macrophage phenotypes are heterogeneous and plastic $[13,14]$. One broad classification distinguishes M1 inflammatory macrophages, which express high IL-12, iNOS and low IL-10, from M2 regulatory macrophages 
which express high IL-10 and low IL-12 [14]. In diseases such as atherosclerosis and malignancy, these polarised macrophage phenotypes make particular contributions to pathogenesis [14]. Resident lamina propria macrophages in health are characterised by inflammatory anergy but enhanced phagocytic and microbicidal capacity [15]. In CD, lamina propria macrophages are more numerous, probably due to recruitment of CD14+ monocytes, and have greater pro-inflammatory cytokine expression [16]. However, the roles of differing macrophage phenotypes in $C D$ pathogenesis are incompletely understood.

The aim of this study was to characterize macrophage phenotypes according to E. coli carriage in mucosal biopsies from patients with $\mathrm{CD}, \mathrm{UC}$ and healthy controls. This may provide insight into potential roles for macrophage phenotypes and E. coli in CD pathogenesis.

\section{Methods}

\section{Study participants}

Patients with IBD were recruited at routine colonoscopy. Diagnoses of $\mathrm{CD}$ and $\mathrm{UC}$ were established by conventional criteria. Asymptomatic healthy controls were recruited at surveillance colonoscopy for previous colorectal polyps or a family history of colorectal cancer. Clinical and demographic data were collected. All participants provided written consent and the study received ethical approval (REC ref. 07/H0804/78).

\section{Mucosal biopsies and biopsy processing}

Biopsies were taken from the colon in healthy controls and from inflamed +/- uninflamed sites (ileal (CD only) or colonic mucosa (CD and UC)) in patients with IBD. In a subgroup of $C D$ patients, paired inflamed and uninflamed biopsies were taken from the same segment of bowel. Endoscopic severity was graded using the simple endoscopic score for CD (SES-CD) [17] and the modified Baron's score for UC [18]. Histological severity was graded from a biopsy adjacent to the study biopsy. Biopsies were snap frozen in liquid nitrogen and stored at $-80{ }^{\circ} \mathrm{C}$. Samples were removed from storage and orientated onto OCT media. $6 \mu \mathrm{m}$ frozen sections were cut onto $1 \mathrm{~mm}$ PALM membrane slides.

\section{Immunodetection of CD68+ macrophages and presence or absence of intracellular $E$. coli}

LP macrophages with or without $E$. coli co-localisation were identified in mucosal biopsies using previously validated immunolabelled CD68+ [19] and E. coli antibodies [20]. A rapid indirect immunostaining protocol was employed for macrophage-specific CD68 (PG M1) to minimize risk of mRNA degradation [21]. CD68 positive cells were detected using a Vectastain ABC-AP kit and a Vector Red chromogenic substrate. Detection of intracellular E. coli was achieved by co-staining CD68+ macrophages with an anti-E. coli polyclonal antibody and labeled with Vector Blue chromogenic substrate. Staining was visualized using a Zeiss axioplan MOT $400 \mathrm{M}$ microscope. CD68+ cells co-localising with anti-E. coli antibody were termed E. coli-laden macrophages (Fig. 1a, b). CD68+ cells without anti-E. coli antibody co-localisation were termed E. coli-unladen macrophages (Fig. 1c).

\section{Laser capture microdissection (LCM) of macrophages and mRNA extraction}

Laser capture was performed using a PALM microsystem. CD68+ only or CD68+/E. coli + cells were isolated under direct microscopic visualisation (Fig. 1d, e) and catapulted onto a PALM adhesive cap. 400-500 E. coli-unladen and, when present, 400-500 E. coli-laden, macrophages were collected per biopsy for sufficient pooled RNA extraction (approximately $35 \mathrm{ng}$ ). RNA extraction was performed using the Cells Direct kit (Invitrogen). Cells were incubated with lysis solution at $75{ }^{\circ} \mathrm{C}$ for 15 mins. Contents were spun down and treated with DNAse1 for 5 mins. The reaction was stopped by adding $25 \mathrm{mM}$ EDTA and kept on ice. RNA was quantified using a Nanodrop spectrophotometer (Thermofisher) and RNA integrity was assessed using an Agilent 2100 Bioanalyser. The range of RIN values was 8.7 9.5 with a cut off of 8.0 for sufficient integrity of RNA [22].

\section{Primer design}

RT-qPCR assays were designed, validated and optimized according to the Minimum Information for Publication of Quantitative Real-Time PCR Experiments (MIQE) guidelines [23]. The mRNA sequences for macrophage activation markers (CD163, iNOS and COX-2) and cytokines (IL-6, IL-8, IL-10, IL-23 and TNF $\alpha$ ) (primer details - Table 1) were identified using the Genbank database. Primers were designed using Beacon Designer, version 7.2, selecting for a primer annealing temperature of $55^{\circ} \mathrm{C}$ and amplicon length of $<100$ bp. Primer and amplicon specificity were checked using Primer-BLAST (http:// www.ncbi.nlm.nih.gov/tools/primer-blast/) and nucleotideBLAST (www.ncbi.nlm.nih.gov/BLAST/) respectively. Target secondary structures and primer/template accessibility were assessed using the MFOLD web server (http:// www.bioinfo.rpi.edu/applications/mfold) using corrections for ionic conditions of $50 \mathrm{nM} \mathrm{Na++}$ and $3 \mathrm{mM} \mathrm{Mg++}$ and a folding temperature of $55{ }^{\circ} \mathrm{C}$. Lyophilised primers were reconstituted in 1xTE buffer to make a stock solution of $100 \mu \mathrm{M} / \mu \mathrm{l}$.

\section{RT-qPCR assays}

RT-qPCR assays were performed using target-specific primer sequences on a Rotor-Gene 6000, with quantification cycles (Cqs) calculated using Corbett software version 1.7 (build 65). Experiments were carried out in duplicate, and 


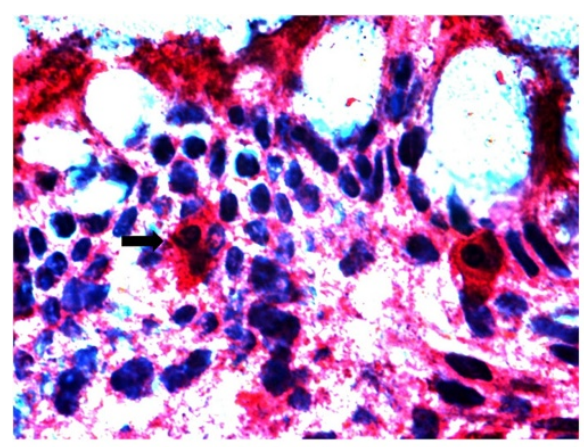

a
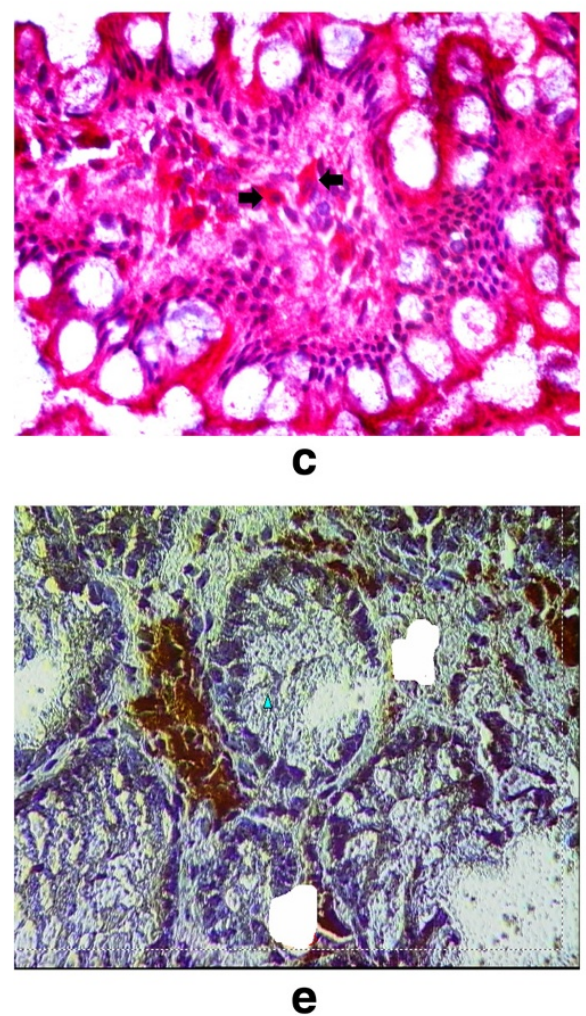

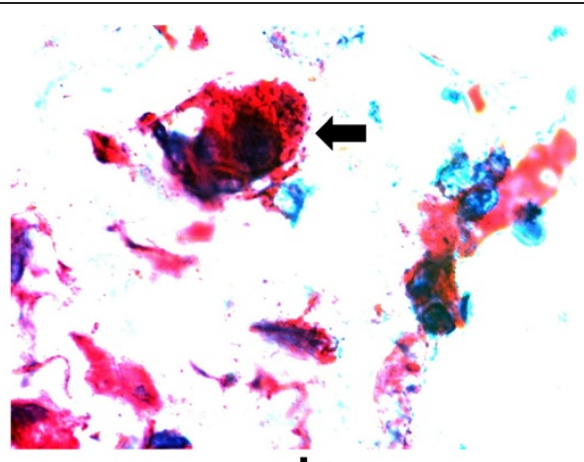

b

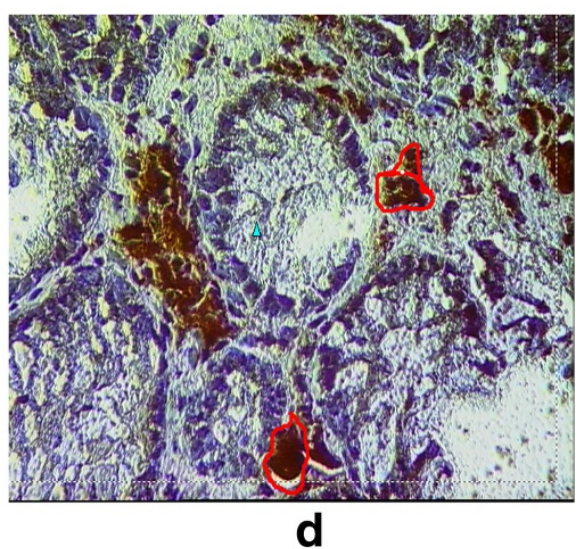

Fig. 1 CD68 staining macrophages (red) can be seen to contain E. coli ( blue ) in the biopsy from a patient with CD ((a) low power ( 40 ), (b) high power (x63), but not in biopsies from a healthy control (x20) (c). Labelled lamina propria macrophages (d) before and (e) after laser capture microdissection

data obtained from replicates yielding a standard deviation of $\mathrm{Cq}$ values of $<0.5$ were used for further analysis.

\section{Quantification and normalisation of data}

mRNA copy numbers were determined from targetspecific standard curves of known concentration (10-fold serial dilutions of RNA extracted from fresh frozen tissue biopsy; $100 \mathrm{ng} / \mu \mathrm{l}-1 \mathrm{pg} / \mu \mathrm{l}$ ) included with every RT-qPCR run. Cq values were plotted against the logarithm of calculated copy numbers and individual target copy numbers were obtained from the linear regression of the standard curve. Data for each target were normalized against fresh biopsy total RNA and expressed as copies of mRNA/ $\mu \mathrm{g}$ total RNA, as recommended [24]. mRNA expression of candidate genes in inflamed CD biopsies was analysed relative to non-inflamed biopsies and controls.

\section{Statistical analysis}

Statistical analysis was performed using SPSS 19.0. Unpaired t-tests and one-way analysis of variance (ANOVA) were used to compare means between 2 groups or greater than 2 groups respectively. Paired t-tests were used for within subject comparisons. Where normality could not be confirmed, non-parametric testing was performed. Fisher's exact test was used to analyse contingency tables. ANCOVA (ANOVA with covariates) was used to 
Table 1 Oligonucleotide primer pairs used for RT-qPCR analysis

\begin{tabular}{lll}
\hline Oligo name & GC $\%$ & Sequence \\
\hline TNFa Sense & 50 & CGAACATCCAACCTTCCAAAC \\
TNFa Anti-Sense & 42 & TGGTGGTCTTGTTGCTAAAGTTC \\
iNOS Sense & 42 & CATCAACAACAATGTGGAGAAAGC \\
iNOS Anti-sense & 55 & TCTGCTGCTTGCTGAGGTTG \\
IL-6 Sense & 48 & GACAGCCACTCACCTCTTCAG \\
IL-6 Anti-sense & 50 & GGAAGGTCAGGTTTTTCTGC \\
IL-8 Sense & 52 & CAGCCACTACAAACAGAGCACTG \\
IL-8 Anti-sense & 48 & CAAAGGGATGACAAGCAGAAAG \\
IL-23 Sense & 54 & GGA CAA CAG TCA GTT CTG CTT GC \\
IL-23 Antisense & 50 & GGA GGC TGC GAA GGA TTT TG \\
IL-10 Sense & 55 & CCAAGACAACACTACTAAGGCTCCTTT \\
IL-10 Antisense & 48 & GCTTCTATATGCTAGTCAGGTA \\
COX-2 Sense & 59 & CTCCTATTATACTAGAGCCCTTCCTC \\
COX-2 Anti-sense & 58 & TITCCAATCTCATTTGAATCAGG \\
CD163 Sense & 48 & AGGGTGATAGAAGAGGCCAACACT \\
CD163 Anti-sense & 50 & TTGCACCGGACAAACTTCATGGC \\
\hline
\end{tabular}

Genbank accession numbers: iNos (L09210), Cox-2 (NM000963), IL-6 (M14584), TNF (M10988), IL-8 (Y00787), IL-10 (M57627), IL-23 (M652720), CD163 (NM0004244)

determine the effect of age on expression of cytokines and markers. $P$ values $<.05$ were considered significant.

\section{Results}

\section{Patients and controls}

Data was analysed from 35 patients with CD, 9 with UC and 18 healthy controls. Mean age was greater in healthy controls (49 years) than in patients with CD (37 years) $(P=.005)$ but not significantly greater than in patients with UC (39 years) $(P=.149)$. Other clinical and demographic details are recorded in Table 2. Biopsy site, and endoscopic and histological severity are recorded in Table 3.

\section{Prevalence of E. coli-laden LP macrophages in CD, UC and healthy controls}

E. coli-laden macrophages were commonly identified in mucosal biopsies from CD patients (25/35 (71\%)), rarely in UC $(1 / 9(11 \%))$ and were not present in any of 18 healthy controls (Table 3 ). The presence of $E$. coli-laden macrophages in $C D$ correlated with endoscopic severity $(P<.001)$ (Fig. 2) but not with other clinical or demographic factors (age, gender, smoking status, disease location, immunomodulation or site of biopsy - data not shown). Six of the $35 \mathrm{CD}$ patients had paired biopsies taken from macroscopically inflamed and uninflamed mucosa. In these 6 patients, E. coli-laden LP macrophages were present in 6/6 inflamed and 3/6 uninflamed biopsies respectively.
Table 2 Clinical and demographic details of study participants

\begin{tabular}{|c|c|c|c|}
\hline & $C D$ & UC & Healthy controls \\
\hline Number of participants & 35 & 9 & 18 \\
\hline Age in years mean (range) & $37(19-65)$ & $39(27-68)$ & $49(23-62)$ \\
\hline Gender (f, m) & 17,18 & 3,6 & 8,10 \\
\hline \multicolumn{4}{|l|}{ Smoking status } \\
\hline Never & 13 & 5 & 10 \\
\hline Current & 7 & 1 & 3 \\
\hline Ex- & 3 & 2 & 2 \\
\hline unknown & 12 & 1 & 3 \\
\hline \multicolumn{4}{|l|}{ Disease distribution } \\
\hline Ileal only & 6 & - & $\mathrm{n} / \mathrm{a}$ \\
\hline Ileo-colonic & 9 & - & $\mathrm{n} / \mathrm{a}$ \\
\hline Colonic only & 20 & 9 & $\mathrm{n} / \mathrm{a}$ \\
\hline \multicolumn{4}{|l|}{ Immunomodulation } \\
\hline Yes & 13 & 4 & 0 \\
\hline No & 22 & 5 & 18 \\
\hline \multicolumn{4}{|l|}{ Anti-TNFa therapy } \\
\hline Yes & 2 & 0 & 0 \\
\hline No & 33 & 9 & 18 \\
\hline
\end{tabular}

\section{Macrophage cytokine and surface marker mRNA expression in CD, UC and healthy controls LP macrophage cytokine mRNA expression in healthy controls is low}

Mean mRNA expression of most cytokines and surface markers (TNF $\alpha$, IL-23, IL-6, IL-8 \& IL-10, iNOS \& COX2 $(P<.001$ for each)) was lower in LP macrophages from healthy controls than from $E$. coli-laden or unladen macrophages from inflamed CD mucosa (Fig. 3(a) to (g)). Mean CD163 mRNA expression in LP macrophages from healthy controls was not significantly different to E. coli-unladen macrophages $(P=.173)$ but lower than E. coli-laden CD macrophages $(P<.001)$ (Fig. $3(\mathrm{~h}))$.

In inflamed CD mucosa, E. coli-unladen macrophages have high proinflammatory cytokine mRNA expression, whereas E. coli-laden macrophages have high IL-10 and CD163 mRNA expression

In inflamed mucosal biopsies from the $25 \mathrm{CD}$ patients in whom both $E$. coli-laden and unladen macrophages were present, E. coli-unladen macrophages had higher mean pro-inflammatory cytokines (TNF $\alpha$, IL-23, IL-6, IL-8) and iNOS expression (Fig. 3(a) - (e), for each; $P<.001$ ) than $E$. coli-laden macrophages (Fig. 3). E. coli-unladen macrophages also expressed higher COX-2 than $E$. coli-laden macrophages (Fig. 3(f) $P<.001)$. Conversely, 
Table 3 Biopsy site and proportion of subjects with E. coli-laden macrophages

\begin{tabular}{llll}
\hline Subject group & CD & UC & $\begin{array}{l}\text { Healthy } \\
\text { controls }\end{array}$ \\
\hline $\begin{array}{l}\text { Subject numbers } \\
\text { Biopsy site }\end{array}$ & 35 & 9 & 18 \\
$\begin{array}{l}\text { rectum } \\
\text { colon (other) }\end{array}$ & 11 & 9 & 18 \\
ileum & 9 & - & - \\
jejunum & 14 & - & - \\
Total & 1 & - & - \\
& $25 / 35(71 \%)$ & $1 / 9(11 \%)$ & $0 / 18(0 \%)$ \\
& $(71 \%)((71 \%)$ & &
\end{tabular}

According to histological severity

$\begin{array}{llll}\text { normal } & 3 / 7 & 0 / 0 & 0 / 18 \\ \text { mild } & 20 / 26 & 0 / 8 & - \\ \text { moderate } & 2 / 2 & 1 / 1 & - \\ \text { severe } & 0 & 0 / 0 & -\end{array}$

According to endoscopic severity score

$\mathrm{CD}$ patients

SES-CD (biopsied segment)

0

$0 / 3$

$1-2$

$3 \quad 6 / 11$

$413 / 15$

$5-7 \quad 6 / 6$

$>7$

SES-CD (total score)

$0 \quad 0 / 3$

$1-2$

$3-4 \quad 8 / 14$

5-8 $9 / 10$

$9-13 \quad 7 / 7$

$>13$

UC patients

Modified Baron score

0

$1 \quad 0 / 8$

$2-1 / 1$

3

patient with jejunal CD - SES-CD total not recorded as no colonoscopy $H C$ healthy controls; UC ulcerative colitis; $C D$ Crohn's disease

E. coli-laden macrophages had higher IL-10 and CD163 expression (Fig. 3(g), (h), for each; $P<.001$ ) than E. coliunladen macrophages. Macrophage mRNA profiles in CD were not associated with any pattern of disease distribution, biopsy site (ileal or colonic), current IM, anti-TNF $\alpha$ therapy or smoking status (data not shown).

\section{E. coli-unladen macrophages are more pro-inflammatory} when E. coli-laden macrophages are present

Mean pro-inflammatory cytokine mRNA expression of E. coli-unladen macrophages was higher in the $25 \mathrm{CD}$ patients in whom E. coli-laden macrophages were also present compared with that in $E$. coli-unladen macrophages in the $10 \mathrm{CD}$ patients' biopsies in which $E$. coliladen macrophages were not present (TNF $\alpha$, IL-23, IL-6, IL-8, iNOS; all $P<.001$ ) (Fig. 3(a) $-(\mathrm{e})$ ).

\section{In UC, E. coli-unladen LP macrophages express elevated} cytokine $M R N A$ and $E$. coli-laden macrophages are rare

Only $1 / 9$ biopsies from patients with UC contained $E$. coli-laden LP macrophages. Mean TNF $\alpha$ expression of $E$. coli-unladen macrophages in UC was not significantly different to that in $E$. coli-unladen macrophages in $C D$ (Fig. 3(a)). Other mean cytokine and surface marker expression in UC E. coli-unladen macrophages varied from CD as follows: IL-6 \& IL-8 not significantly different to CD E. coli-laden macrophages; iNOS \& CD163, intermediate between CD E. coli-laden and unladen; and COX2 not significantly different to healthy controls (IL-23 and IL-10 not measured for UC) (Fig. 3).

\section{Macrophage phenotypes in paired inflamed and uninflamed biopsies in $C D$}

E. coli-laden macrophages expressed a similar phenotype in inflamed and uninflamed mucosa (Fig. 4(a) - (d)). Conversely, E. coli-unladen macrophages from uninflamed mucosa expressed much lower cytokine and surface marker mRNA levels than in inflamed mucosa (for all $P<.001$ except CD163 $P=$ NS) (Fig. 4(a) - (d)). These were at levels closer to, and in some cases, indistinguishable from controls.

\section{Discussion}

In this study, E. coli-laden lamina propria macrophages were identified commonly in $\mathrm{CD}$, rarely in $\mathrm{UC}$ and not at all in healthy control mucosal biopsies. In CD, there were distinct macrophage phenotypes in relation to the carriage of E. coli.

\section{Intra-macrophage $E$. coli in $\mathrm{CD}$}

We found that $E$. coli can be identified within LP macrophages in most CD patients using immunohistochemistry with an anti-E. coli specific polyclonal antibody. This concurs with previous studies that demonstrate E. coli within LP macrophages in CD using immunohistochemistry [5] and FISH [25] or within granulomas using LCM and nested PCR [10]. In future studies, further confirmation of the presence of $E$. coli within lamina propria macrophages in $C D$ could be achieved using an alternative technique such as 16S rRNA PCR. Additionally, the presence of other bacteria within macrophages could be determined 


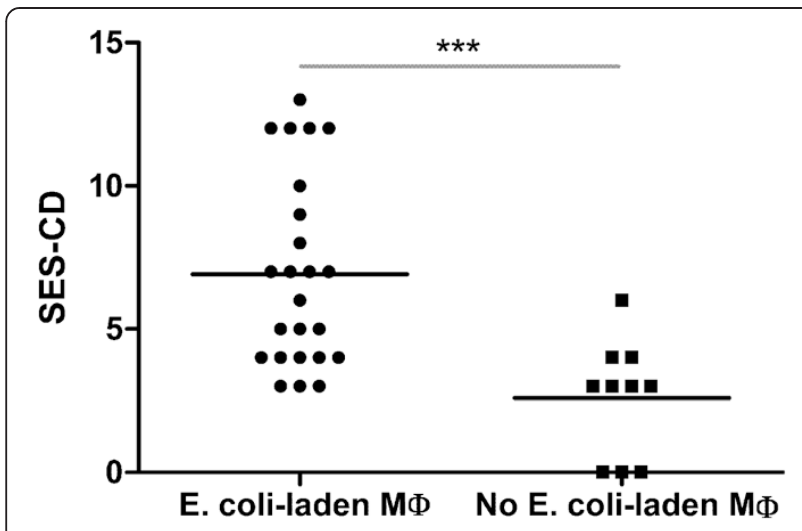

Fig. 2 Higher median endoscopic severity score for CD patients with $E$. coli-laden LP macrophages compared with those without E. coli-laden LP macrophages at biopsy $(P<.001$, Mann-Whitney test). SES-CD; simplified endoscopic score for Crohn's disease, $M \varnothing ;$ macrophages

by extracting DNA from laser-captured macrophages, performing bacterial $16 \mathrm{~S}$ rDNA sequencing and comparing any $16 \mathrm{~S}$ rDNA sequences present to database reference bacterial sequences [26].

It is possible that the presence of intramacrophage $E$. coli in CD results from successful adherence to and invasion of the mucosa by AIEC, with subsequent survival and replication within LP macrophages. Certainly, in vitro, AIEC have been shown to possess properties that might facilitate this process $[8,9]$. However, it is also possible that an innate defect of bacterial killing by LP macrophages contributes to $E$. coli persistence within macrophages, a concept which is also supported by the presence of macrophage cytokine defects and mutations in bacterial handling genes in $\mathrm{CD}[1,27,28]$. We did not determine whether intra-macrophage $E$. coli were AIEC in our study because these $E$. coli cannot be distinguished morphologically. However, using culture of biopsies with gentamicin protection to isolate intracellular $E$. coli from the mucosa in $C D$, we have found that only a minority of intracellular isolates have adherent and invasive properties in vitro [12].

The lower prevalence (1/9) of E. coli-laden macrophages in UC is in keeping with previous reports of lower intramucosal bacteria in UC than $\mathrm{CD}[6,29]$. Meanwhile, the absence of $E$. coli-laden macrophages in all 18 healthy subjects illustrates that the mucosal immune system prevents bacterial persistence within the LP in health.

\section{Measurement of macrophage surface marker \& cytokine mRNA expression using real time RT-PCR}

Real-time RT-PCR can provide a sensitive and accurate measurement of mRNA when performed in accordance with the MIQE guidelines. Normalisation of mRNA data remains a contentious issue. House-keeping genes such as GAPDH and $\beta$-Actin have been proposed as being stable appropriate reference genes for normalisation. Prior to initiation of this study, we performed preliminary experiments using GAPDH and $\beta$-Actin and found that these reference genes were unstable in these inflamed tissue samples. We also have previously published data demonstrating that internal reference genes may not be appropriate for normalisation of qPCR data for mRNA, especially when derived from tissue biopsies [30, 31]. However, the use of total RNA for normalisation has been demonstrated to be valid [24] and produce quantification results that are biologically relevant [30] as long as certain criteria are met $[32,33]$. These are that the RIN is above 8 (which can be considered perfect total RNA for downstream applications [22]) which was confirmed using the Agilent 2100 Bioanalyzer in our study, and the use of small amplicons which minimise the variability caused by RNA degradation. Hence, our decision to use total RNA for normalisation in this study is valid, especially as we are reporting very large and characteristic differences in expression of mRNA for a range of cytokines between Crohn's disease, healthy controls and UC that are not consistent with chance RNA degradation.

\section{Characteristic LP macrophages according to E. coli carriage in $C D$}

In healthy controls, cytokine and surface marker mRNA expression in macrophages from uninflamed colonic mucosa were low, in keeping with previous data on intestinal macrophages in health [34]. In inflamed CD mucosa, there was clear differentiation of macrophage cytokine and surface marker profiles according to $E$. coli carriage ( $E$. coli-unladen; higher proinflammatory cytokines (TNF $\alpha$, IL-23, IL-6, IL-8) and iNOS, and E. coli-laden; higher IL-10 and CD163, both characteristic features of regulatory macrophages). The phenotype of $E$. coli-unladen macrophages in CD is consistent with that of recently recruited CD14+ macrophages, which secrete high TNFa and IL-23 and are more numerous in active CD [16]. It is likely that these activated macrophages contribute significantly to inflammation and recruitment of other proinflammatory cells important in CD pathogenesis such as Th17 cells [16]. E. coli-unladen macrophages in uninflamed mucosa from $\mathrm{CD}$ patients with active disease expressed very low cytokine mRNA levels, similar to healthy controls, and are therefore likely to be inactive resident LP macrophages [15].

The observed phenotype (high IL-10, lower TNFa) of E. coli-laden macrophages in $\mathrm{CD}$ might represent an appropriate regulatory response to microbial encroachment, or may facilitate $E$. coli persistence, and thus contribute to pathogenesis. Supporting the former supposition, the immunoregulatory role of macrophages secreting IL-10 

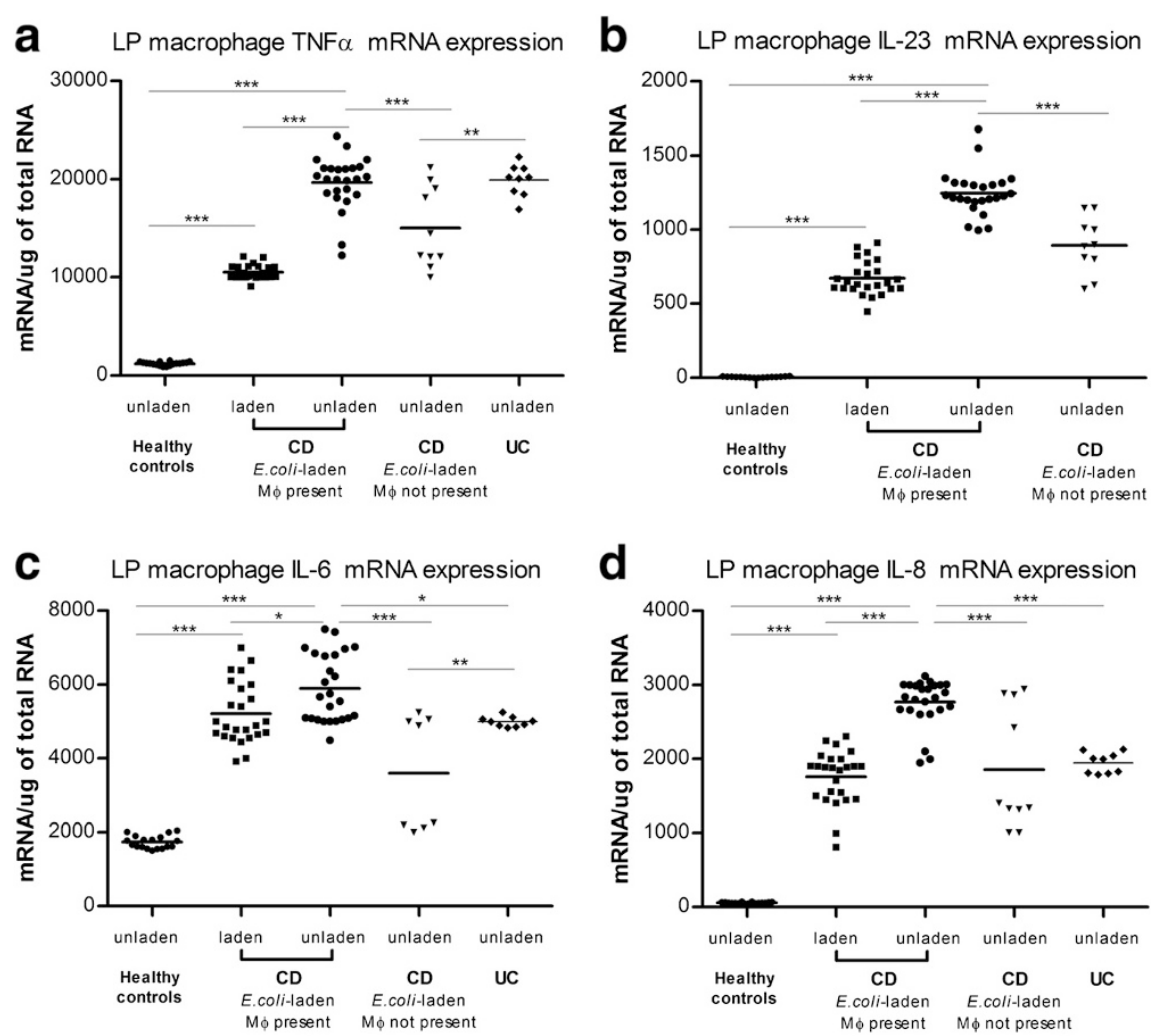

e LP macrophage iNOS mRNA expression

f LP macrophage COX2 mRNA expression
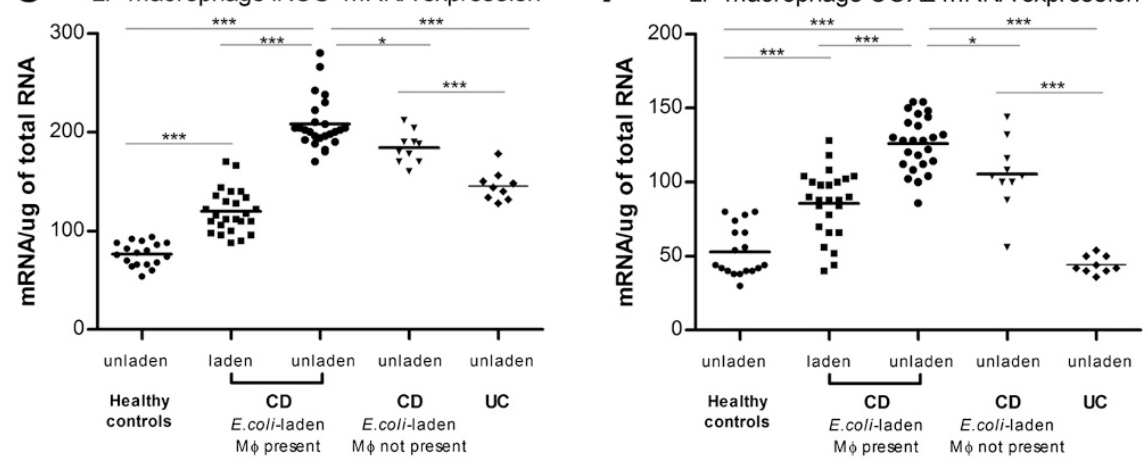

9 LP macrophage IL-10 mRNA expression

h LP macrophage CD163 mRNA expression
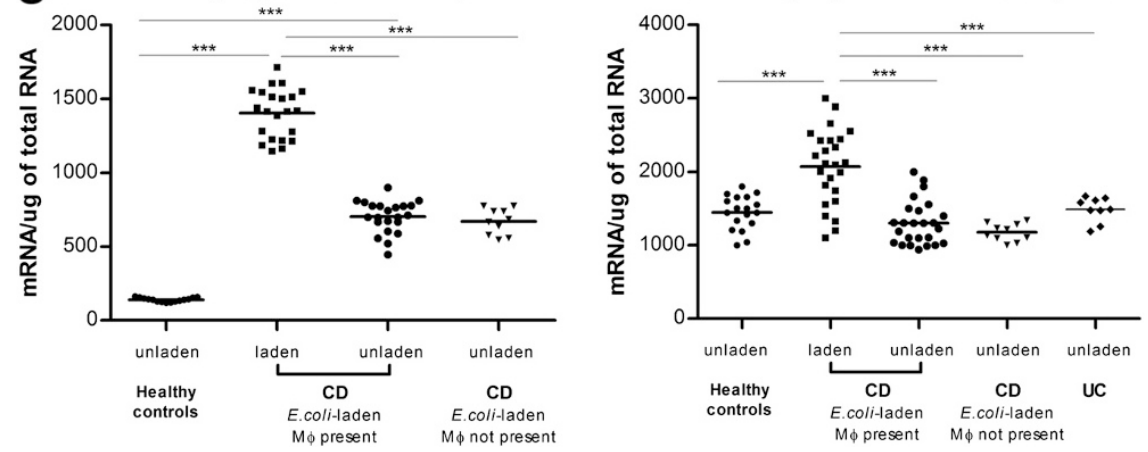

Fig. 3 (See legend on next page.) 
(See figure on previous page.)

Fig. 3 Cytokine and surface marker mRNA levels in healthy controls, CD patients with E. coli laden macrophages, CD patients without E. coli laden macrophages and UC patients. Fig. 3(a) From left to right: Healthy controls $(n=18)$; LP macrophages have low TNFa mRNA expression. Inflamed mucosal biopsies from CD patients with both $E$. coli-laden and unladen macrophages present $(n=25)$; $E$. coli-unladen macrophages had higher mean TNFa mRNA expression $(P<.001)$ than $E$. coli-laden macrophages. CD patients without $E$. coli-laden macrophages $(n=10)$; TNFa mRNA expression of $E$. coli-unladen macrophages were lower than in $E$. coli-unladen macrophages from the $25 \mathrm{CD}$ patients in whom both $E$. coli-laden and unladen were present $(P<.001)$. UC patients $(n=9)$; E. coli-unladen LP macrophages showed elevated TNFa mRNA levels. Fig 3(b) to (f): Expression of other proinflammatory cytokines (IL-23, IL-6, IL-8) and iNOS is similar to the pattern of TNFa expression in samples from each subject group. Fig. 3 (g) Expression of COX2 is similar to the pattern of TNFa in each group but is lower in UC than in CD. In distinction to the pattern of TNFa expression, Fg. $3(\mathbf{g})$ and $3(\mathbf{h})$ demonstrate that IL-10 and CD-163 mRNA expression are higher in E. coli-laden than E. coli-unladen macrophages in CD (for each; $P<.001$ ). ${ }^{*} P<.05$, ${ }^{* *} P<.01,{ }^{* * *} P<.001$. Comparisons of means made with one-way ANOVA and Games-Howell post-hoc pair-wise comparisons. There was no UC data for IL-23 or IL-10. Only one UC patient had E. coli-laden macrophages (cytokine data not shown)

is well documented [35]. However, IL-10 also facilitates intracellular persistence of numerous microorganisms [35], possibly due to inhibition of autophagy [36]. Interestingly, in Whipple's disease, Tropheryma Whipplei accumulate in duodenal macrophages which express a similar phenotype to the E. coli-laden macrophages in this study (high IL-10, CD163) which is thought to facilitate their persistence [37].

E. coli-laden macrophages were present and also expressed a high IL-10 phenotype in uninflamed mucosa in 3 of the $6 \mathrm{CD}$ patients with active disease in whom paired inflamed/uninflamed biopsies were taken. This raises the possibility that $E$. coli access the mucosa at an early stage of $C D$ pathogenesis rather than as a consequence of a disrupted inflamed mucosa. Of note, the presence of $E$. coli-laden macrophages correlated with endoscopic severity and higher pro-inflammatory cytokine mRNA expression of $E$. coli-unladen macrophages. This highlights further the dilemma of cause and effect as this may either be because the presence of E. coli in LP macrophages causes intestinal inflammation or that their presence is merely a consequence of inflammation.

In UC, E. coli-unladen macrophage cytokine and surface marker expression were elevated often to similar levels as in $\mathrm{CD}$, however COX-2 mRNA was substantially lower in
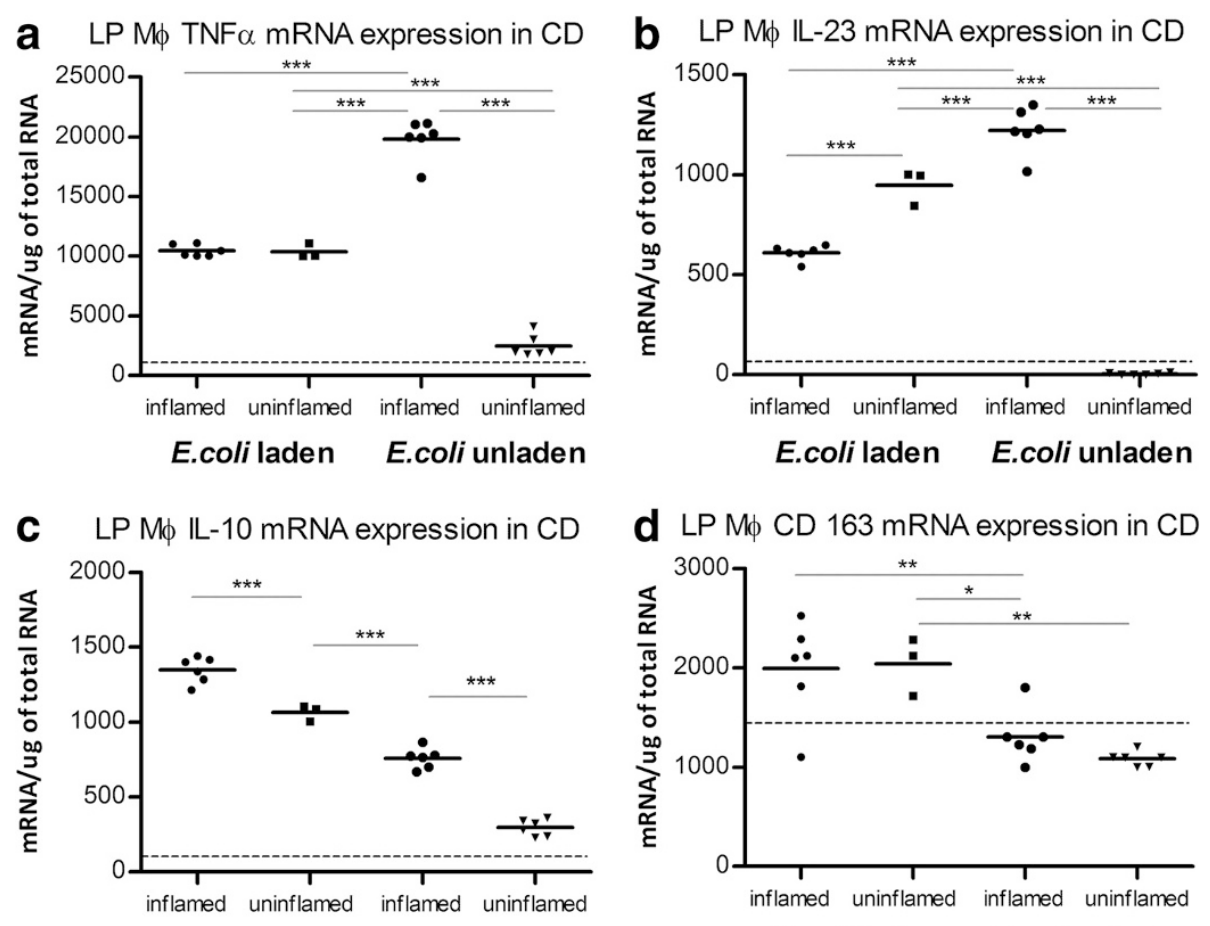

E.coli laden E.coli unladen

d $L P M \phi C D 163$ mRNA expression in CD

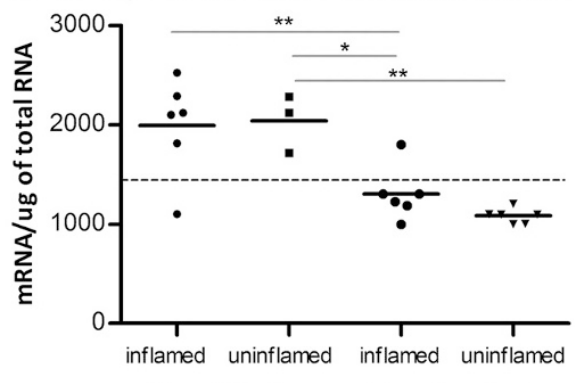

E.coli laden E.coli unladen

Fig. 4 E. coli-laden macrophages express a similar phenotype in inflamed and uninflamed tissue high IL-10 (c), CD163 (d), lower TNFa (a) and IL-23 (b). E. coli-unladen macrophages express a proinflammatory phenotype in inflamed tissue high TNFa (a), and IL-23 (b), lower IL-10 (c), CD163 (d) but in uninflamed tissue express cytokine mRNA expression nearer to that of healthy controls (dotted line = healthy control median mRNA expression). $M \varnothing=$ macrophage. ${ }^{*} P<.05,{ }^{* *} P<.01,{ }^{* * *} P<.001$. Paired t tests used for within subjects statistical analysis. Presence or absence of inflammation determined by macroscopic appearance at endoscopy 
UC. This may be in keeping with a recent Danish study that reported the association of a COX-2 gene polymorphism (A-1195G variant allele) with UC but not CD [38]. The authors hypothesised that the mutation, which is associated with low COX-2 activity, may lead to increased UC susceptibility because of reduced prostaglandin synthesis as prostaglandins regulate mucosal inflammation. It would be of interest to correlate the prevalence of this mutation with macrophage COX-2 expression in UC in future work.

\section{Conclusions}

This study provides novel insights into the presence of intramacrophage $E$. coli and lamina propria macrophage phenotypes in relation to the presence or absence of intracellular E. coli in Crohn's disease. Further work is required to determine the pathogenic significance of these macrophage subtypes in $\mathrm{CD}$. Clarification of whether E. coli-laden macrophages are attempting to ameliorate inflammation or whether they contribute to microbial persistence and disease pathogenesis will also be important. This may lead to therapies aimed at manipulation of macrophage phenotypes $[13,14]$ and renewed interest in the use of antibiotics that target intracellular E. coli for the treatment of CD [39].

\section{Abbreviations \\ CD: Crohn's disease; UC: Ulcerative colitis; HC: Healthy controls; f: female; m: male; ex-: ex-smoker; TNFa: Tumour necrosis factor-alpha; n/a: not applicable; MØ: Macrophage; SES-CD: Simplified endoscopic score for Crohn's disease; AIEC: Adherent-invasive E. coli.}

\section{Competing interests}

The authors have no financial or non-financial competing interests to declare.

\section{Authors' contributions}

*TRE \& NBR contributed equally to this paper: study concept and design; acquisition of data; analysis and interpretation of data; drafting of the manuscript; statistical analysis. NBR performed the LCM of macrophages. $\mathrm{BNH}$ : study concept and design; acquisition of data; analysis and interpretation of data; drafting of the manuscript. REH: technical support with LCM work; acquisition of data. GCP \& KT: acquisition of data; analysis and interpretation of data. SAB: study design; acquisition of data; analysis and interpretation of data. $N J P, J H-T, J B, L P, A B$ \& CGM: critical revision of the manuscript for intellectual content. JDS: study concept and design; acquisition of data; analysis and interpretation of data; drafting of the manuscript; obtained funding. All authors read and approved the final manuscript.

\section{Acknowledgements}

We acknowledge the National Institute for Health Research (NIHR) Biomedical Research Centre award to Guy's \& St. Thomas' National Health Service (NHS) Trust, King's College London. We also acknowledge the Broad Foundation, USA who contributed funding to this work by an Inflammatory Bowel Disease grant. N.B.R was funded by the Broad Foundation and B.N.H \& G.C.P were funded by Foundation for Allergy Information and Research, UK. The authors would like to thank Professor Anthony d'Apice and Professor Peter Cowan, Immunology Research Centre, St Vincent's Hospital, Melbourne, Australia, for their critical review of this manuscript.

\section{Author details}

'Diabetes and Nutritional Sciences Division, King's College London, Franklin Wilkins Building, 150 Stamford Street, London SE1 9NH, UK. 'Department of Gastroenterology, Guy's and St Thomas' NHS Foundation Trust, St Thomas'
Hospital, London SE1 7EH, UK. ${ }^{3}$ Department of Medical and Molecular Genetics, King's College London School of Medicine, Guy's Hospital, London SE1 9RT, UK. "Blizard Institute, Barts and the London School of Medicine and Dentistry, Queen Mary University of London, Whitechapel, London E1 1BB,

UK. ${ }^{5}$ Department of Medicine, University of Melbourne, Melbourne, Australia. ${ }^{6}$ Postgraduate Medical Institute, Faculty of Medical Science, Anglia Ruskin University, Chelmsford, Essex, UK.

Received: 22 January 2015 Accepted: 18 June 2015

Published online: 03 July 2015

\section{References}

1. Smith AM, Rahman FZ, Hayee B, et al. Disordered macrophage cytokine secretion underlies impaired acute inflammation and bacterial clearance in Crohn's disease. J Exp Med. 2009;206:1883-97.

2. Homer CR, Richmond AL, Rebert NA, et al. ATG16L1 and NOD2 interact in an autophagy-dependent antibacterial pathway implicated in Crohn's disease pathogenesis. Gastroenterology. 2010;139:1630-41. 1641 e1631-1632.

3. Sartor RB. Does Mycobacterium avium subspecies paratuberculosis cause Crohn's disease? Gut. 2005;54:896-8.

4. Lamps LW, Madhusudhan KT, Havens JM, et al. Pathogenic Yersinia DNA is detected in bowel and mesenteric lymph nodes from patients with Crohn's disease. Am J Surg Pathol. 2003;27:220-7.

5. Liu Y, van Kruiningen $H J$, West $A B$, et al. Immunocytochemical evidence of Listeria, Escherichia coli, and Streptococcus antigens in Crohn's disease. Gastroenterology. 1995;108:1396-404.

6. Martin HM, Campbell BJ, Hart CA, et al. Enhanced Escherichia coli adherence and invasion in Crohn's disease and colon cancer. Gastroenterology. 2004;127:80-93.

7. Darfeuille-Michaud A, Boudeau J, Bulois P, et al. High prevalence of adherentinvasive Escherichia coli associated with ileal mucosa in Crohn's disease. Gastroenterology. 2004;127:412-21.

8. Glasser AL, Boudeau J, Barnich N, et al. Adherent invasive Escherichia coli strains from patients with Crohn's disease survive and replicate within macrophages without inducing host cell death. Infect Immun. 2001;69:5529-37.

9. Baumgart M, Dogan B, Rishniw M, et al. Culture independent analysis of ileal mucosa reveals a selective increase in invasive Escherichia coli of novel phylogeny relative to depletion of Clostridiales in Crohn's disease involving the ileum. ISME J. 2007;1:403-18.

10. Ryan P, Kelly RG, Lee G, et al. Bacterial DNA within granulomas of patients with Crohn's disease-detection by laser capture microdissection and PCR. Am J Gastroenterol. 2004:99:1539-43.

11. Mylonaki M, Rayment NB, Rampton DS, et al. Molecular characterization of rectal mucosa-associated bacterial flora in inflammatory bowel disease. Inflamm Bowel Dis. 2005;11:481-7.

12. Elliott TR, Hudspith BN, Wu G, Cooley M, Parkes GC, Quiñones B, et al. Quantification and characterization of mucosa-associated and intracellular Escherichia coli in inflammatory bowel disease. Inflamm Bowel Dis. 2013;19:2326-38

13. Gratchev A, Kzhyshkowska J, Kothe K, et al. Mphi1 and Mphi2 can be re-polarized by Th2 or Th1 cytokines, respectively, and respond to exogenous danger signals. Immunobiology. 2006;211:473-86.

14. Mosser DM, Edwards JP. Exploring the full spectrum of macrophage activation. Nat Rev Immunol. 2008;8:958-69.

15. Smith PD, Smythies LE, Shen R, et al. Intestinal macrophages and response to microbial encroachment. Mucosal Immunol. 2011;4:31-42.

16. Kamada N, Hisamatsu T, Okamoto S, et al. Unique CD14 intestinal macrophages contribute to the pathogenesis of Crohn disease via IL-23/ IFN-gamma axis. J Clin Invest. 2008;118:2269-80.

17. Daperno M, D'Haens $G$, Van Assche $G$, et al. Development and validation of a new, simplified endoscopic activity score for Crohn's disease: the SES-CD. Gastrointest Endosc. 2004;60:505-12.

18. Baron JH, Connell AM, Lennard-Jones JE. Variation between observers in describing mucosal appearances in proctocolitis. Br Med J. 1964;1:89-92.

19. Falini B, Flenghi L, Pileri S, et al. PG-M1: A new monoclonal antibody directed against a fixative-resistant epitope on the macrophage-restricted form of the CD68 molecule. Am J Pathol. 1993;142:1359-72.

20. Berry RE et al. Urothelial cultures support intracellular bacterial community formation by uropathogenic Escherichia coli. Infect Immun. 2009;77:2762-72. 
21. Trogan E, Fisher EA. Laser capture microdissection for analysis of macrophage gene expression from atherosclerotic lesions. Methods Mol Biol. 2005;293:221-31. Review.

22. Fleige $\mathrm{S}, \mathrm{Pfaffl} M W$. RNA integrity and the effect on the real-time $\mathrm{qRT}$-PCR performance. Mol Aspects Med. 2006;27(2-3):126-39.

23. Bustin SA, Benes V, Garson JA, et al. The MIQE quidelines: minimum information for publication of quantitative real-time PCR experiments. Clin Chem. 2009;55(4):611-22.

24. Bustin SA. Quantification of mRNA using real-time reverse transcription PCR (RT-PCR): trends and problems. J Mol Endocrinol. 2002;29(1):23-39.

25. Rayment N, Mylonaki M, Hudspith B, et al. Co-localisation of E. coli with macrophages in lamina propria in patients with active inflammatory bowel disease (IBD). Gut. 2003:52:A13.

26. Woo PC, Lau SK, Teng JL, et al. Then and now: use of 165 rDNA gene sequencing for bacterial identification and discovery of novel bacteria in clinical microbiology laboratories. Clin Microbiol Infect. 2008;14(10):908-34.

27. Elliott TR, Hudspith BN, Rayment NB et al. Defective macrophage handling of Escherichia coli in Crohn's disease. J Gastroenterol Hepatol. 2015 Mar 23. doi: 10.1111/jgh.12955. [Epub ahead of print]

28. Prescott NJ, Fisher SA, Franke A, et al. A nonsynonymous SNP in ATG16L1 predisposes to ileal Crohn's disease and is independent of CARD15 and IBD5. Gastroenterology. 2007;132:1665-71.

29. Sasaki M, Sitaraman SV, Babbin BA, et al. Invasive Escherichia coli are a feature of Crohn's disease. Lab Invest. 2007:87:1042-54.

30. Tricarico $C$ et al. Quantitative real-time reverse transcription polymerase chain reaction: normalization to rRNA or single housekeeping genes is inappropriate for human tissue biopsies. Anal Biochem. 2002;309(2):293-300.

31. Dheda $\mathrm{K}$ et al. Validation of housekeeping genes for normalizing RNA expression in real-time PCR. Biotechniques. 2004;37(1):112. -4, 116, 118-9.

32. Bustin SA, Benes V, Nolan T, Pfaffl MW. Quantitative real-time RT-PCR - a perspective. J Mol Endocrinol. 2005;34:597-601.

33. Nolan T, Hands RE, Bustin SA. Quantification of mRNA using real-time RT-PCR Nat Protoc. 2006;1:1559-82.

34. Smythies LE, Sellers M, Clements $\mathrm{RH}$, et al. Human intestinal macrophages display profound inflammatory anergy despite avid phagocytic and bacteriocidal activity. J Clin Invest. 2005;115:66-75.

35. Mege JL, Meghari $\mathrm{S}$, Honstettre A, et al. The two faces of interleukin 10 in human infectious diseases. Lancet Infect Dis. 2006;6:557-69.

36. Park HJ, Lee SJ, Kim SH, et al. IL-10 inhibits the starvation induced autophagy in macrophages via class I phosphatidylinositol 3-kinase (PI3K) pathway. Mol Immunol. 2011:48:720-7.

37. Moos V, Schmidt C, Geelhaar A, et al. Impaired immune functions of monocytes and macrophages in Whipple's disease. Gastroenterology. 2010;138:210-20.

38. Andersen V, Nimmo E, Krarup HB, et al. Cyclooxygenase-2 (COX-2) polymorphisms and risk of inflammatory bowel disease in a Scottish and Danish case-control study. Inflamm Bowel Dis. 2011;17:937-46.

39. Subramanian S, Roberts CL, Hart CA, et al. Replication of colonic Crohn's disease mucosal escherichia coli isolates within macrophages and their susceptibility to antibiotics. Antimicrob Agents Chemother. 2008:52:427-34.

\section{Submit your next manuscript to BioMed Central and take full advantage of:}

- Convenient online submission

- Thorough peer review

- No space constraints or color figure charges

- Immediate publication on acceptance

- Inclusion in PubMed, CAS, Scopus and Google Scholar

- Research which is freely available for redistribution 